\title{
Computer-controlled displays of bending motions
}

\author{
WARREN D. CRAFT, THEODORE J. PAYNE, and JOSEPH S. LAPPIN \\ Vanderbilt University, Nashville, Tennessee
}

This paper describes a physical model and the mathematical equations specifying the dynamics of a bending line. A computer program is also described that will control real-time displays of a line of points in three-dimensional space and collect psychophysical data from human observers discriminating between different bending motions.

Wallach and his coworkers (Wallach \& O'Connell, 1953; Wallach, O'Connell, \& Neisser, 1953; Wallach, Weisz, \& Adams, 1956) reported about 30 years ago on a phenomenon they called the kinetic depth effect (KDE) and demonstrated that the human visual system is capable of correctly abstracting three-dimensional structure from two-dimensional images of rigid motion. Since then, the KDE has stimulated much research and thought. An important recent finding is that human observers are apparently capable of correctly abstracting three-dimensional structure from two-dimensional images of nonrigid motion (e.g., Jansson, 1977; Jansson \& Johansson, 1973; Jansson \& Runeson, 1977). Growing theoretical interest in this phenomenon stems from the fact that the perception of kinetic depth from nonrigid motion challenges contemporary theories of the KDE that have been based on the structural constraints associated with rigid motion. Although theories have usually assumed rigidity, vision evidently does not.

Very little psychophysical or theoretical research on the perception of nonrigid motion is available, however. One major reason for that lack is the relative inaccessibility of information governing the shapes and motions of significantly nonrigid forms. As a consequence, it is difficult to produce accurate or realistic stimulus displays and even more difficult to program a computer to display and control such stimuli.

We are currently investigating the optical information that visually specifies three-dimensional structure from nonrigid motion. To do this, we needed to develop a computer-controlled display of bending motion that could be systematically manipulated by a variety of control parameters, such as elasticity, magnitude of bend, length, temporal oscillation frequency, three-dimensional orientation, number of points, and so forth. This paper presents our solution of this problem and describes a program for displaying a bending line of points. (Although we have not yet generalized our efforts to the bending of a surface, Koenderink \& van Doorn, 1986, have recently described a model involving discrete approximations to a bending surface.)

This work was supported by NIH Grant EY05926. The authors' mailing address is Department of Psychology, Vanderbilt University, Nashville, TN 37240.

\section{MATHEMATICAL MODEL}

To produce a physically valid version of a bending, elastic material, we took as a model a cantilever beam with an end couple-that is, a beam anchored at one end and free at the other, with an end couple produced by parallel forces at each end, in opposite directions, in the plane of the elastic curve. Such a beam, in bent and unbent positions, is illustrated in Figure 1, as conventionally depicted in engineering texts.

The problem, then, is to find an equation that describes the dynamics of an elastic material undergoing bending deformations. Typically, studies in the mechanics of materials assume only small deflections and, thus, are concerned only with the vertical deflection, assuming no horizontal deflection in Figure 1. We need to derive an equation without such an assumption.

The bending of a three-dimensional object produces a surface with positive stress and strain (it would be compressed as would the unseen upper surface of the beam in Figure 1) and a surface with negative stress and strain (it would be under tension as would the unseen bottom surface of the beam in Figure 1). Parallel to and midway between those two surfaces is a neutral surface, for which stress and strain are both zero. When undergoing a bending deformation, that neutral surface is isometric in time, undergoing no compression and suffering no tension.

Introductory elastic theory shows that the curvature of a neutral surface, at a given point in time and a given distance along the curve, may be expressed as

$$
\frac{1}{\varrho}=\frac{M}{E I},
$$

where $\varrho$ is the radius of curvature, $M$ the bending moment, $E$ the modulus of elasticity, and $I$ the moment of inertia of the cross-section about its neutral axis. In the International System of Units (SI units), $\varrho$ would be expressed in meters, $M$ as force $\times$ length in newton-meters or $\left(\mathrm{kg} \cdot \mathrm{m}^{2}\right) / \mathrm{s}^{2}, E$ in newtons per square meter $\left(\mathrm{N} / \mathrm{m}^{2}\right)$, and $I$ in $m^{4}$. Thus, the curvature is directly proportional to the bending moment, $M$, which is a measure of the energy or torque at each point along the beam; in this case, $M$ increases in proportion to the distance along the beam from the fixed end. The curvature is inversely propor- 


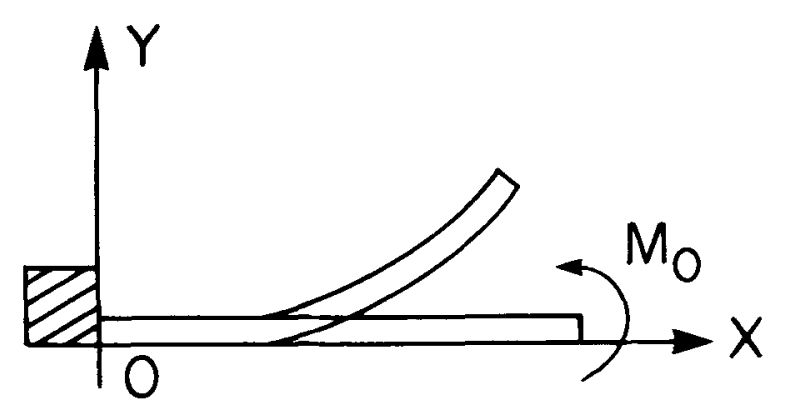

Figure 1. Two-dimensional image of cantilever beam with end couple, in bent and unbent positions.

tional to the elastic modulus, $E$, or stiffness of the beam, as well as inversely proportional to $I$, the moment of inertia of the cross section, which depends upon both the cross-sectional area of the beam and the distance from the fixed end of the beam. Dimensional analysis of Equation 1 shows that the three physical characteristics $E, I$, and $M$, involving three fundamental physical measures (mass, length, and time), combine to yield a onedimensional measure of the curvature, $1 / \varrho$, in $\mathrm{m}^{-1}$. Thus, $E, I$, and $M$ can be combined in a single "bend parameter," and in subsequent development we will find it convenient to do so, maintaining the distance, $x$, from the fixed end of the beam as a separate control parameter.

To describe the temporal change in curvature associated with the bending motion, we will allow the bend parameter to change sinusoidally with time, as the restoring force of a swinging pendulum varies sinusoidally with time.

According to elementary calculus, the curvature of a plane curve may be expressed as

$$
\frac{1}{\varrho}=\frac{d^{2} y / d x^{2}}{\left[1+(d y / d x)^{2}\right]^{3 / 2}},
$$

where $y(x)$ represents the function of the curve. Combining Equations 1 and 2 gives us

$$
\frac{M}{E I}=\frac{d^{2} y / d x^{2}}{\left[1+(d y / d x)^{2}\right]^{3 / 2}} .
$$

Unfortunately, attempting to produce a general equation for the elastic curve by integration of Equation 3 leads to an elliptic integral. A different formulation, however, has been found to yield a very good approximation to the elastic curve.

First, we envision the beam as being deflected only in the vertical direction. Thus, it is clear that considerable deflection can occur before the slope $d y / d x$ is significantly greater than unity. With that in mind, we develop a set of parametric equations describing the elastic curve. The first of these equations will be an expression for $y^{\prime}$, the ordinate of a point on the curve in terms of its original position in $x$, and the second will be a similar expression for the abscissa, $x^{\prime}$, for a point on the curve in terms of its original position $x$. By initially assuming no horizon- tal deflection, and assuming that $(d y / d x)^{2}$ is fairly small compared to unity, Equation 3 becomes

$$
\frac{d^{2} y^{\prime}}{d x^{2}}=\frac{M}{E I}
$$

Integrating twice and solving for the constants of integration using the following boundary conditions:

$$
\begin{aligned}
y^{\prime} & =0 \text { when } x=0, \\
\frac{d y^{\prime}}{d x} & =0 \text { when } x=0,
\end{aligned}
$$

we arrive at the following equation for the ordinate of a point on the elastic curve:

$$
y^{\prime}=\frac{1}{2} \frac{M_{0}}{E I} x^{2},
$$

where the bending moment $M_{o}$ for each point is given by

$$
M_{o}=(\text { Force })(x)=F \cdot x .
$$

Combining Equations 6 and 7, we arrive at

$$
y^{\prime}=\frac{1}{2} \frac{F}{E I} x^{3}=\frac{1}{2} K x^{3} \text {. }
$$

In Equation 8, the physical characteristics $E, I$, and $M$ have been combined into a single "bend parameter," $K$.

The $x$-coordinate, $x$ ', of a point on the curve may be calculated after considering a formula given by Roark (1943) for the change $\Delta H$ in the length of the horizontal projection of the curve as it bends, which states that

$$
\Delta H=\frac{1}{2} \int_{0}^{x}\left(\frac{d y}{d x}\right)^{2} d x
$$

where $d y / d x$ is derived from Equation 8. Letting $\Delta H=$ $x-x^{\prime}$, we have

$$
\begin{aligned}
x-x^{\prime} & =\frac{1}{2} \int_{0}^{x} \frac{9}{4} K^{2} x^{4} d x \\
& =\frac{9}{8} K^{2}\left[\frac{1}{5} x^{5}\right]_{0}^{x} \\
& =0.225 K^{2} x^{5},
\end{aligned}
$$

which gives us

$$
x^{\prime}=x-0.225 K^{2} x^{5} .
$$

Now, combining Equations 8 and 10, we have for a general equation of the elastic curve

$$
x^{\prime}=A-0.225 K^{2} A^{5},
$$

where $A=\left(2 y^{\prime} / K\right)^{1 / 3}$.

Because of the approximate nature of the solution, a systematic distortion of the elastic curve is introduced in the form of stretching of the interpoint distance. To cor- 


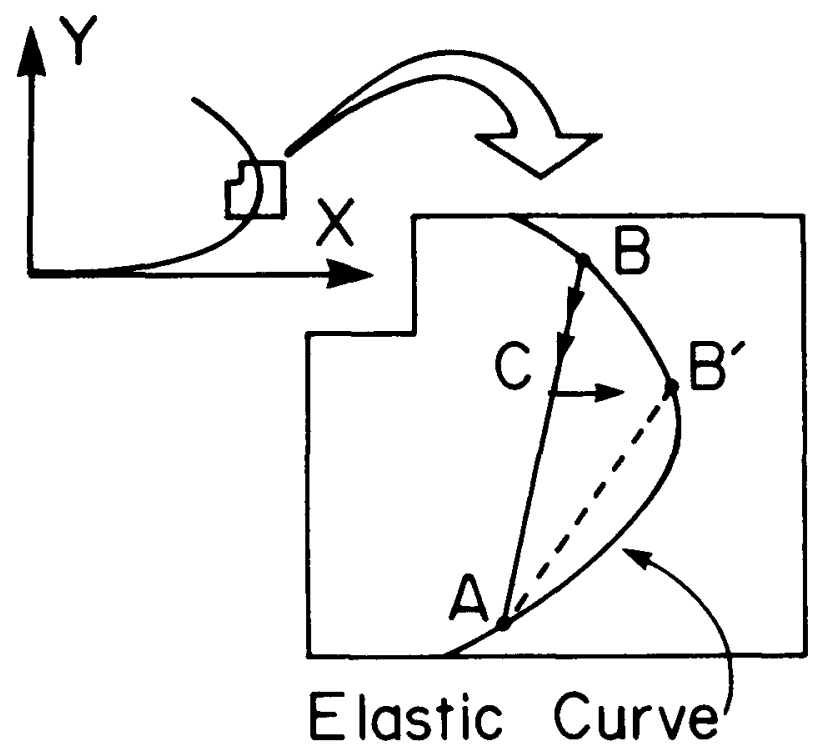

Figure 2. Iterative scheme for correcting interpoint distance to original distance after bending (see text).

rect this, we take the shape of the curve as essentially correct, and we implement an iterative correction scheme to reposition points along the curve at the original interpoint distance, $S$. As illustrated in Figure 2, point $A$ is a previously corrected point, while point $B$ is a point yet to be corrected. Point $B$ is moved along the chord $\overline{A B}$ to position $C$, where the distance $A C$ is equal to $S$. The point is then placed back on the curve by taking the ordinate at $C$ and plugging it into Equation 11 above, giving the point shown as $B^{\prime}$ in Figure 2. The process is repeated until $1.000<A B^{\prime} / S<1.001$.

Bending motion is produced by stepping through a number of frames equidistant in time, with each frame representing a value of the bend parameter $K$, which varies sinusoidally with time between any desired $K_{\max }$ and $-K_{\max }$.

Different types of bends may be produced, then, by changing the way the bend parameter varies along the length of the beam at any particular time. For a "standard bend," $K$ is kept constant along the beam at any particular time.
An alternative case is the "linearly increasing bend," where $K$ varies along the beam at time $t$ from $K=\mathrm{K}_{t_{\min }}$ at the base to $K=K_{t_{\max }}$ at the free end, where $K_{t_{\min }}$ and $K_{t_{\max }}$ are the minimum and maximum $K$ at the time $t$.

A step function bend is created by allowing $K$ to assume two discrete values along the curve, changing at some discontinuity point $D$. The final display of one frame of points is generated as suggested in Figure 3. First, a frame is generated for a standard bend using the value of $K_{1}$ at time $t$, where $K_{1}$ is the value of the bend parameter from the base to the discontinuity point $D$, and $K_{2}$ is the value for the beam from point $D$ to the free end. Second, a similar frame is generated using $K_{2}$. The slope at point $D$ is calculated for each curve, and from them, the angle between the two is calculated and stored. Finally, a single curve is created by combining the bottom section of the $K_{1}$ curve with the top section of the $K_{2}$ curve, but first rotating the $K_{2}$ curve so that the slope of its tangent to point $D$ is equal to that of the tangent to point $D$ in the $K_{1}$ curve.

\section{PROGRAM}

\section{Input}

The program is controlled by a series of nested menus, and allows the user to create as many as 100 unique stimulus patterns by choosing one or more values for each of the following: length and number of points of a beam, phase-frequency sets, and three-dimensional orientations of plane of motion; the number of unique stimuli, then, would be the product of the number of beam lengths, the number of phase-frequency sets, the number of orientations, and the number of bend functions in the task. The user also assigns values to the following: trial duration time, number of trials, number of practice trials, number and value of simulated viewing distances, and interocular separation.

\section{Output}

For each task, an equal number of stimuli are generated for each bend function. The order of trials is random, and the stimuli are directed to two CRT screens as
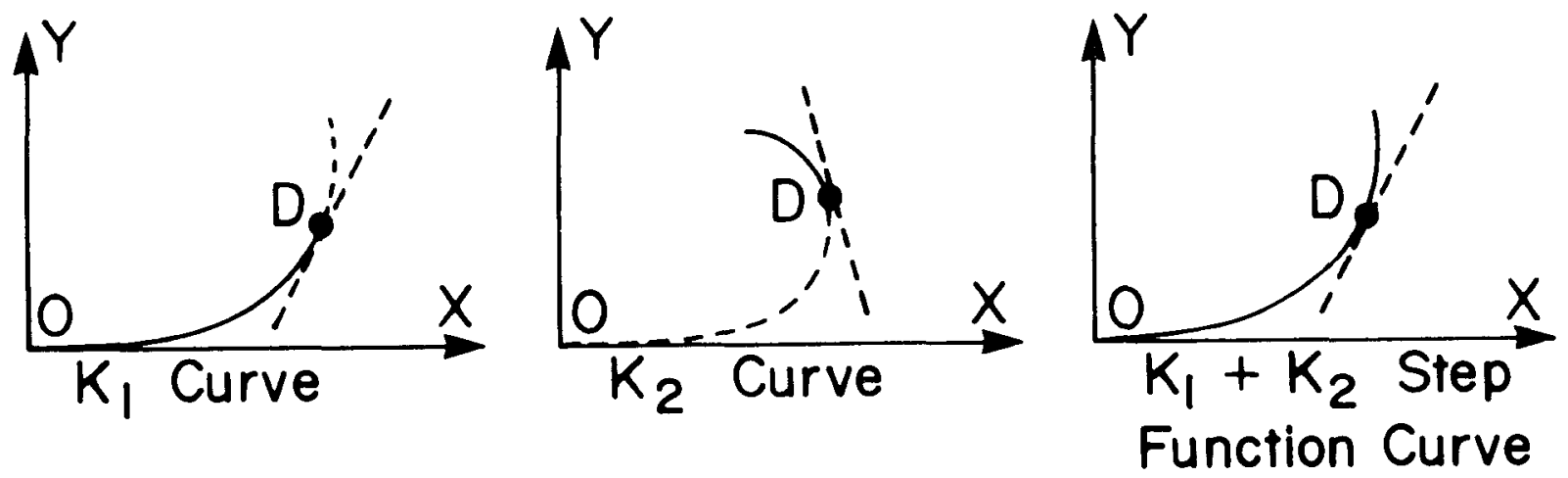

Figure 3. Creation of a "step function bend." Bottom section of $K_{1}$ curve is joined to top section of $K_{2}$ curve after rotation of $K_{2}$ curve (see text). 


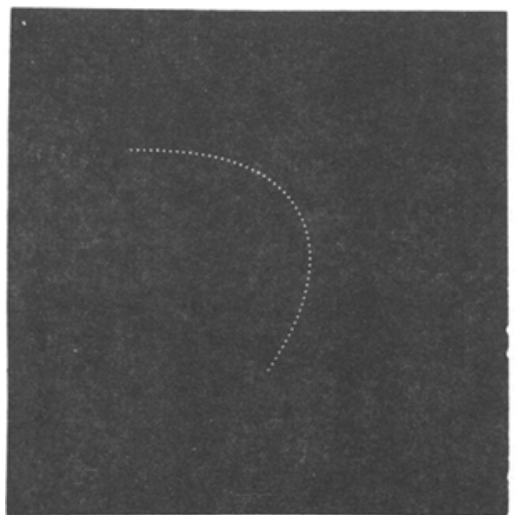

A

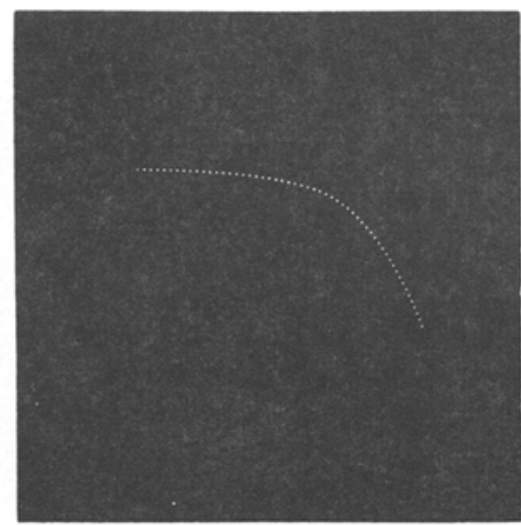

B

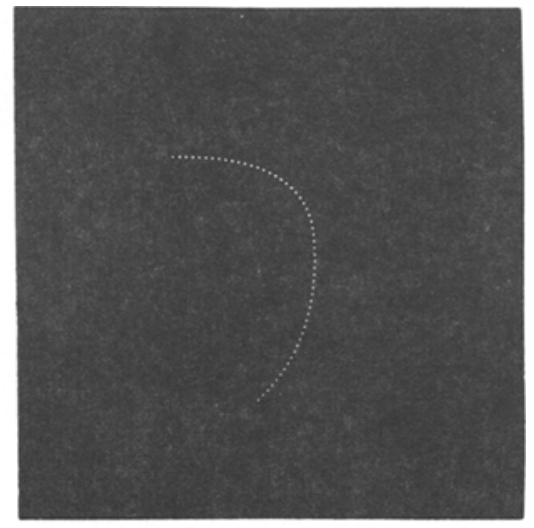

C

Figure 4. Photographs of CRT displays of (A) a "standard bend," (B) a "step function bend" with free end more flexible than secured end, and (C) a "step function bend" with secured end more fexible than free end.

a part of a stereoscopic display system. The derived equations along with the choice of three-dimensional orientation allow three-dimensional placement of the stimulus; projection onto the two-dimensional screen is accomplished using standard mathematical equations. Figure 4 shows CRT displays of three functions: (A) a "standard bend," (B) a "step function bend" with the free end more flexible than the secured end, and (C) a "step function bend" with the secured end more flexible than the free end. Subjects begin a trial by pushing a button on a keypad before them, at which time a beam of points would begin bending, oscillating back and forth. After each trial, the program collects the subject's response, and after all trials are finished, a trial-by-trial record of the session is printed on a line printer. The program then prints out a confusion matrix and, in the case of a $2 \times 2$ matrix, calculates and prints out $-\ln \eta$, a measure of discriminal distance corrected for response bias. ${ }^{1}$

\section{Language, Requirements, and Limitations}

The program was written in PDP-11 FORTRAN (FORTRAN IV) with macro subroutines in PDP Macro-11. Implemented on an RT-11 (version 4.0) operating system on an LSI 11/73 microcomputer, the compiled program requires approximately $65 \mathrm{~K}$ bytes of disk space, but because of overlays needs only $44 \mathrm{~K}$ bytes of RAM.

Without virtual memory, the average intertrial interval is approximately $5 \mathrm{sec}$. Using virtual memory to store all point information prior to a block of trials eliminates the need for that extended intertrial interval.

\section{Availability}

A listing of the program contains extensive commentary to aid the experienced user wishing to explore and make modifications. A listing of the program (or a copy) may be obtained free of charge from the authors. If a copy is desired, please send a single-sided, double-density, 8-in diskette in a reusable diskette mailer. Program users with questions or suggestions may reach the authors by phone at 615-322-6067.

\section{REFERENCES}

JANsson, G. (1977). Perceived bending and stretching motions from a line of points. Scandinavian Journal of Psychology, 18, 209-215.

JANSson, G., Johansson, G. (1973). Visual perception of bending motion. Perception, 2, 321-326.

Jansson, G., \& Runeson, S. (1977). Perceived bending motion from a quadrangle changing form. Perception, 5, 595-600.

Koenderink, J. J, \& VAN Doorn, A. J. (1986). Depth and shape from differential perspective in the presence of bending deformations. Journal of the Optical Society of America, 2, 242-249.

LuCE, R. D. (1963). Detection and recognition. In R. D. Luce, R. R. Bush, \& E. Galanter (Eds.), Handbook of mathematical psychology (pp. 103-189). New York: Wiley.

RoARK, R. J. (1943). Formulas for stress and strain. New York: McGraw-Hill.

Wallach, H., \& O'ConNell, D. N. (1953). The kinetic depth effect. Journal of Experimental Psychology, 45, 205-217.

Wallach, H., O'ConNell, D. N., \& Neisser, U. (1953). The memory effect of visual perception of three-dimensional form. Journal of Experimental Psychology, 45, 360-368.

Wallach, H., Weisz, A., ADams, P. (1956). Circles and derived figures in rotation. American Joumal of Psychology, 69, 48-59.

\section{NOTE}

1. The measure of accuracy, from Luce's (1963) choice theory, is defined by the equation:

$$
-\ln \eta=\left(\frac{1}{2}\right) \ln \left\{\frac{\left[N\left(R_{1} \mid S_{1}\right)+0.5\right]\left[N\left(R_{2} \mid S_{2}\right)+.05\right]}{\left[N\left(R_{2} \mid S_{1}\right)+0.5\right]\left[N\left(R_{1} \mid S_{2}\right)+.05\right]}\right\},
$$

where the parentheses enclose the subject's response, $R$, to the particular stimulus, $S$, actually presented; 1 and 2 represent the two bend functions being discriminated; and $N()$ represents the number of trials for which the designated response was made for the designated stimulus. Thus, for example, the first set of parentheses in the numerator on the right side is the number of trials in which a subject correctly identified the bend function stimulus 1 . This measure corrects for response bias and has the mathematical property of a distance measure. The measure is similar to the signal detection measure, $d^{\prime}$. 